\title{
Colorless FSK Demodulation and Detection With Integrated Fabry-Pérot-Type SOA/REAM
}

\author{
Bernhard Schrenk, Student Member, IEEE, Jose A. Lazaro, Member, IEEE, Christophe Kazmierski, and \\ Josep Prat, Member, IEEE
}

\begin{abstract}
A receiver for optical frequency modulated signals, based on colorless demodulation and detection, is presented. An integrated combination of semiconductor optical amplifier (SOA) and reflective electroabsorption modulator (REAM) is designed to obtain a comb-like spectral detection function, replacing the typically required costly frequency discrimination filter. The spectral properties of the designed SOA/REAM chip are discussed and the reception of a 10-Gb/s optical frequency modulated downstream signal is demonstrated, proving the applicability as receiving optical subsystem in the customer premises equipment of fiber-to-thehome access networks with wavelength reuse for upstream transmission and no downstream crosstalk.
\end{abstract}

Index Terms-Optical fiber communication, optical modulation, optical resonators, optical signal detection.

\section{INTRODUCTION}

$\mathbf{F}$ IBER-TO-THE-HOME networks are a promising solution for the access segment. Their continuous migration demands the customer premises equipment to provide high data rate service delivery, by retaining its cost-efficiency at the same time. Especially passive optical network (PON) offers low operational and capital expenditures, due to its fully passive fiber plant and the optical network units (ONUs) that are free of wavelength-specific components such as filters or light sources [1]. With its mass deployment, the ONU is determining the cost-efficiency of the access network, and has to be kept, therefore, as simple as possible. This especially requires integrated solutions for its receiving and transmitting subsystems.

Since the customer density of PONs depends on the spectral efficiency once wavelength-division-multiplexing (WDM) techniques are integrated to enhance the capacity of the access network, full-duplex transmission with wavelength reuse for the upstream becomes important. Unfortunately, simple modulation

Manuscript received February 25, 2010; revised April 07, 2010; accepted April 18, 2010. Date of publication May 03, 2010; date of current version June 11, 2010. This work was supported by the European EURO-FOS NoE, by the FP7 SARDANA project, by the Spanish MICINN TEC2008-01887 project, and by the FPU program.

B. Schrenk, J. A. Lazaro, and J. Prat are with the Department of Signal Theory and Communication, Technical University of Catalonia, Barcelona 08034, Spain (e-mail: jprat@tsc.upc.edu).

C. Kazmierski is with the Alcatel-Thales III-V Laboratory, Joint Laboratory of Alcatel-Lucent Bell Laboratories France and Thales Research and Technology, Marcoussis 91461, France (e-mail: christophe.kazmierski@3-5lab.fr).

Color versions of one or more of the figures in this letter are available online at http://ieeexplore.ieee.org.

Digital Object Identifier 10.1109/LPT.2010.2049014
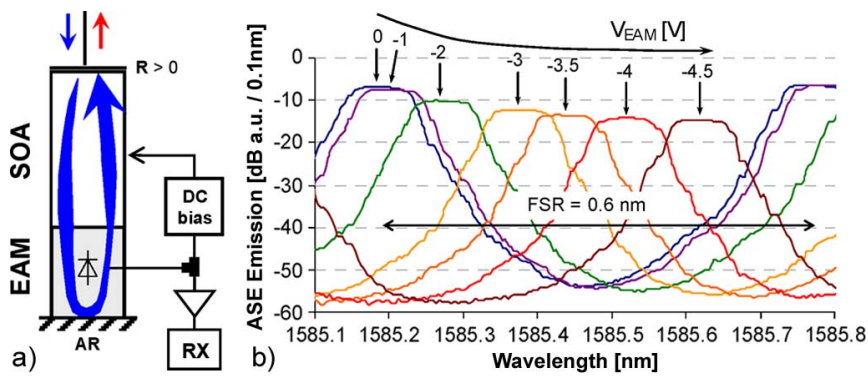

Fig. 1. (a) Scheme of the integrated colorless FSK demodulator and detector, and (b) the obtained comb-like ASE spectrum for an EAM voltage from 0 to $-4.5 \mathrm{~V}$, an SOA current of $100 \mathrm{~mA}$, and a temperature of $22^{\circ} \mathrm{C}$.

formats, such as amplitude-shift keying (ASK) with reduced extinction ratio (ER) for the downstream, suffer in their remodulation quality due to severe crosstalk from the downstream to the upstream. As a result, complex techniques for downstream pattern suppression are required [2]. On the contrary, orthogonal modulation formats for down- and upstream are advantageous. However, for a cost-efficient implementation of the ONU, they are compromised by the complexity of the downstream receiver for the phase-modulated signal, and the desired colorless design that prevents the use of a wavelength-specific filter in front of the photodetector for frequency-shift keying (FSK) [3], [4].

As a solution to this, we propose to use an integrated combination of semiconductor optical amplifier (SOA) and reflective electroabsorption modulator (REAM) [5] to perform the desired functionalities of FSK demodulation and detection for the downstream, which in turn, in combination with an additional reflective modulator, allows to remodulate the constant power FSK downstream signal with ASK upstream data.

\section{COlORLESS FSK Demodulation AND Detection}

Colorless demodulation of FSK signals can be practically performed by a photodetector and a preceding optical filter with a comb-like transmission function and a controlled spectral periodicity. Such a comb filter can be obtained with a travellingwave SOA that is left without antireflection (AR) coating on its facets, which leads to a wavelength-specific gain ripple with Fabry-Pérot spectrum.

Besides, a highly negative biased EAM offers its capability as detector with large electrooptical bandwidth. Together, SOA and EAM enable a single SOA/REAM chip to work as an integrated colorless FSK demodulator and detector [6], as sketched in Fig. 1(a), once the incident signal is adjusted to the comb (or vice versa). 

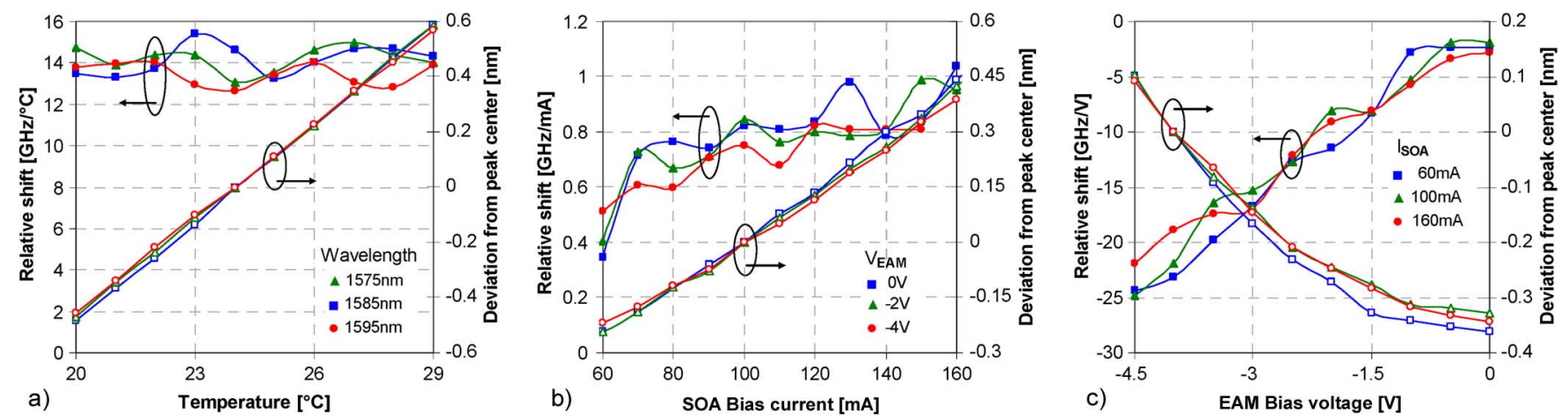

Fig. 2. Induced shifts for the comb, measured at $1586 \mathrm{~nm}$, for (a) variation of the temperature for an SOA bias of $100 \mathrm{~mA}$ and an EAM bias of $-4 \mathrm{~V}$, (b) variation of the SOA bias at a temperature of $22{ }^{\circ} \mathrm{C}$, and (c) variation of the EAM bias at a temperature of $22{ }^{\circ} \mathrm{C}$.

The ripple in the transfer function $P_{\text {det }} / P_{\text {in }}$ for the detection inside the folded active Fabry-Pérot cavity, is derived [7] with

$$
\frac{P_{\mathrm{det}}}{P_{\mathrm{in}}}=\frac{1}{1-R} \frac{(1-R)^{2} G_{\mathrm{soa}} L_{\mathrm{eam}}}{\left(1+R G_{\mathrm{soa}} L_{\mathrm{eam}}\right)^{2}+4 R G_{\mathrm{soa}} L_{\mathrm{eam}} \sin ^{2}\left(\frac{\omega}{\mathrm{FSR}}\right)}
$$

where $R$ is the facet reflectivity, $G_{\text {soa }}$ and $L_{\text {eam }}$ the gain and loss of the SOA and the EAM section, $\omega$ the light frequency, and FSR the free spectral range. The latter is given by the lengths and refractive indices of the SOA and EAM sections, and the passive waveguide.

The EAM section works as a detector inside the cavity, when it is operated in a drop-and-continue mode [8]. In this way, a part of the signal is passed back to the SOA to form the comb.

The input facet of the waveguide that leads to the SOA section was cleaved without angle and aligned to a tapered fiber that is used as fiber pigtail. A chip length of $610 \mu \mathrm{m}$ and the difference in the refractive index between the semiconductor material and the air leads to an FSR of $0.6 \mathrm{~nm}$ and a finesse of 6.3 for typical operation points, as shown in Fig. 1(b). This is in agreement with the grid specification used for WDM, since at least one peak of the comb can be found in each $100-\mathrm{GHz}$ channel.

A spectral shift of the comb derives from variations in the carrier density, which are in turn caused by changes in the input signal power, the SOA and EAM bias, and the temperature. This can be seen in the spectrum of the amplified spontaneous emission (ASE) in Fig. 1(b) for a variation of the EAM bias voltage. Fig. 2 shows the induced shifts around a certain operation point, when different parameters are slightly changed. The relative shifts are thereby related to small perturbations in the altered parameter around the point of operation, and the peak centers of the plots are defined for $24^{\circ} \mathrm{C}, 100 \mathrm{~mA}$, and $-4 \mathrm{~V}$, respectively. The operation point itself is given by the temperature and the SOA and EAM bias.

The shifts can be advantageously used to align the comb to the incident data signal. As can be seen in Fig. 2(a), the shift with temperature is $\sim 14 \mathrm{GHz} /{ }^{\circ} \mathrm{C}$, regardless of the operation point. Variations of the SOA bias, shown in Fig. 2(b), lead to a shift in the order of $0.8 \mathrm{GHz} / \mathrm{mA}$ at a bias current of $100 \mathrm{~mA}$, which slightly depends on the operation point. For a change in the EAM bias, the shift is not linear anymore due to the electrorefractive nature. It strongly depends on the bias point of

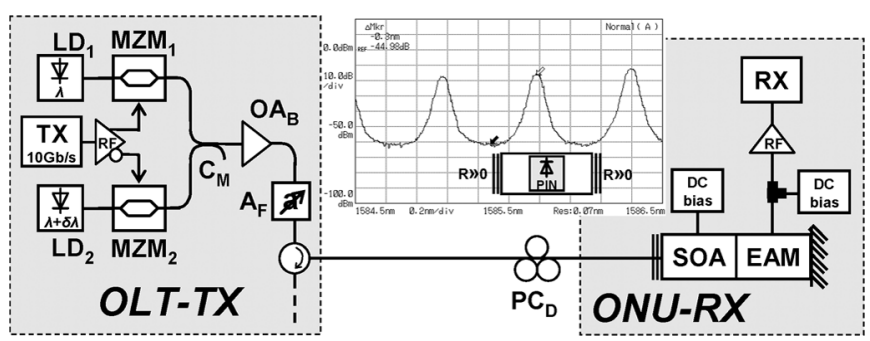

Fig. 3. Experimental setup for the proof of FSK downstream detection. The inset shows the equivalent functional scheme of the FSK detector, and the measured ASE spectrum of the SOA/REAM with a scale of $10 \mathrm{~dB} / \mathrm{div}$ and $0.2 \mathrm{~nm} / \mathrm{div}$.

the EAM, as can be seen in Fig. 2(c), and varies from -2.5 to $-25 \mathrm{GHz} / \mathrm{V}$.

To evaluate the performance of the FSK downstream detection, two complementary ASK modulated signals were used. The detected signal was thereby centered at one of the peaks of the comb, while another, separated from the first by the spacing $\delta \lambda$, corresponding to the frequency deviation of continuousphase FSK signals, is located between two peaks and determines the crosstalk distortion for the detection. The constant signal power of this FSK signal provides a possibility for ASK remodulation to embed upstream data onto the incident downstream.

The FSK modulator (Fig. 3), based on two differentially driven Mach-Zehnder modulators $\left(\mathrm{MZM}_{1}, \mathrm{MZM}_{2}\right)$, provided an ER of $\sim 13 \mathrm{~dB}$ for each of the two ASK signals, while no transients were observed at the bit edges after passing the 50/50 coupler $\left(C_{M}\right)$ that combines the ASK signals. The remaining ER of the FSK signal was $<0.5 \mathrm{~dB}$, giving a good approximation for a constant power for the downstream. An erbium-doped fiber amplifier (EDFA) at the output of the optical line terminal (OLT) compensates for the losses of the FSK modulator. The signal powers and optical signal-to-noise ratios were $4.4 \mathrm{dBm}$ and $44 \mathrm{~dB}$ after the OLT booster. Although this OLT transmitter may not be a cost-effective solution and does not provide a continuous-phase FSK signal, it is suitable for the proof of concept of the proposed ONU.

The FSK detector was characterized in terms of FSK-to-ASK conversion penalty and colorless operation. Due to the remaining polarization sensitivity of the SOA/REAM chip, a polarization controller $\left(\mathrm{PC}_{D}\right)$ was inserted between the OLT 

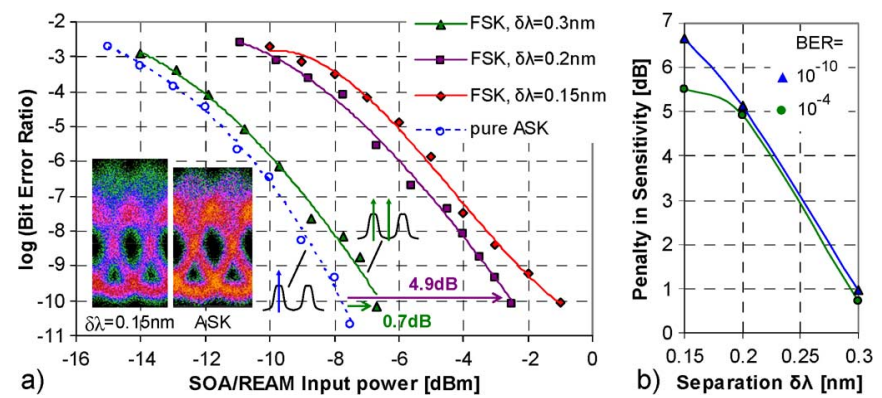

Fig. 4. BER curves and penalty for FSK demodulation when creating a constant signal power downstream signal.

and the ONU. A variable attenuator $\left(A_{F}\right)$ is used for measurements of the bit-error ratio (BER). The optimum bias point for REAM and SOA, operated at a temperature of $22^{\circ} \mathrm{C}$, was found with $-4.1 \mathrm{~V}$ and $80 \mathrm{~mA}$, respectively. The latter was kept low to avoid lasing effects that would appear due to the cleaved chip facet.

The influence of the spacing $\delta \lambda$ on the FSK detection is shown in Fig. 4. For the pure ASK downstream, consisting of a single signal from laser diode LD1, which is aligned to a peak of the comb as sketched in the inset in Fig. 4, a sensitivity of $-7.7 \mathrm{dBm}$ is obtained at a BER of $10^{-10}$ for a $10-\mathrm{Gb} / \mathrm{s}$ signal with a pseudorandom bit sequence (PRBS) of length $2^{31}-1$. For an FSK signal and the optimum case with $\delta \lambda=\mathrm{FSR} / 2=0.3 \mathrm{~nm}$, meaning a placement of the second carrier between two peaks, the penalty that is suffered from applying a constant downstream signal power by adding the second ASK signal is $\sim 1 \mathrm{~dB}$. This low FSK-to-ASK demodulation penalty confirms a good contrast in the comb spectrum, which is sufficiently high to provide an FSK-to-ASK converted signal with a high enough ER. For a smaller separation of $\delta \lambda=0.15 \mathrm{~nm}=\mathrm{FSR} / 4$, the penalty increases up to $6.7 \mathrm{~dB}$. This high penalty is attributed to the low finesse of the FSK detector. For the implementation in a PON with deployed fiber, a trade-off would have to be made between the reception penalty that derives from a reduced contrast due to a closer spacing $\delta \lambda$, and the dispersion penalty that grows with larger spectral widths (i.e., wider spacings $\delta \lambda$ ) of the FSK downstream. Alternatively, a redesign of the chip could lead to a reduced FSR and, therefore, to a smaller required spacing $\delta \lambda$.

Since an EAM is used as signal detector and the SOA section of the chip is used for establishing a cavity, an additional preamplifier would be required at the ONU to increase the sensitivity further. However, considering full-duplex upstream transmission, this additional optical amplifier, which might be an SOA, can be reused for boosting the upstream signal [6].

Fig. 5 shows the proof for a colorless operation of $\delta \lambda=$ $0.3 \mathrm{~nm}$ for different operating wavelengths in a range from 1572 to $1592 \mathrm{~nm}$. The sensitivity at a low BER of $10^{-10}$ is quite independent of the operating wavelength, and deviates by $1.4 \mathrm{~dB}$. Since the gain spectrum of the SOA/REAM, which was designed for the $L$-band, had a roll-off in its gain around $1595 \mathrm{~nm}$, longer wavelengths were not evaluated.

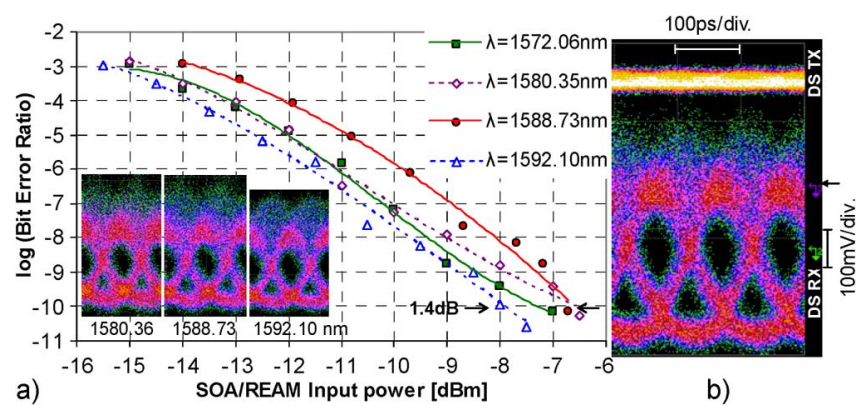

Fig. 5. (a) BER curves showing the colorless FSK demodulation. (b) Eye diagrams for the transmitted (DS TX) and detected (DS RX) downstream after the OLT transmitter and after demodulation, for a wavelength of $1572.06 \mathrm{~nm}$, with an ER of the DS TX of $0.35 \mathrm{~dB}$ (the reference is indicated by the arrow). The scale is $100 \mathrm{mV} / \mathrm{div}$ for both eye diagrams.

\section{CONCLUSION}

An integrated solution for colorless demodulation and detection of FSK signals, based on an SOA/REAM with a comblike transmission function, has been evaluated. The tunability of the comb, achieved via small deviations in the SOA and EAM bias, is suitable for compensating temperature drifts and for aligning the comb to incident data signal, considering wavelength grids that are considered for next-generation PONs. Reception of $10-\mathrm{Gb} / \mathrm{s}$ FSK downstream signals has been shown to suffer from a penalty of $<1 \mathrm{~dB}$ when compared to a reception of an ASK downstream signal, proving the functionality as FSK-to-ASK converter. No strong dependence on the operating wavelength was observed inside the spectral gain region of the SOA/REAM chip. Full-duplex transmission in PONs is left to be analyzed.

\section{REFERENCES}

[1] L. G. Kazovsky, W. Shaw, D. Gutierrez, N. Cheng, and S. Wong, "Nextgeneration optical access networks," J. Lightw. Technol., vol. 25, no. 11, pp. 3428-3442, Nov. 2007.

[2] J. H. Yu, N. Kim, and B. W. Kim, "Remodulation schemes with reflective SOA for colorless DWDM PON," J. Opt. Netw., vol. 6, pp. 1041-1054, Aug. 2007.

[3] N. Genay, P. Chanclou, T. Duong, N. Brochier, and E. Pincemin, "Bidirectional WDM/TDM-PON access networks integrating downstream $10 \mathrm{Gbit} / \mathrm{s}$ DPSK and upstream $2.5 \mathrm{Gbit} / \mathrm{s}$ OOK on the same wavelength," in Proc. ECOC'O6, Cannes, France, Sep. 2006, Paper Th.3.6.6.

[4] J. J. Martinez, J. I. Garces, A. Lopez, A. Villafranca, J. C. Aguado, and M. Losada, "Novel WDM-PON architecture based on a spectrally efficient IM-FSK scheme using DMLs and RSOAs," J. Lightw. Technol., vol. 26, no. 3, pp. 350-356, Feb. 1, 2008.

[5] A. Garreau et al., "10 Gbit/s amplified reflective electroabsorption modulator for colorless access networks," IEEE Proc. Indium Phosphide Rel. Mater., pp. 168-170, May 2006.

[6] B. Schrenk, J. A. Lazaro, C. Kazmierski, and J. Prat, "Colourless FSK/ASK optical network unit based on a Fabry Pérot type SOA/REAM for symmetrical $10 \mathrm{~Gb} / \mathrm{s}$ WDM-PONs," in Proc. ECOC'09, Vienna, Austria, Sep. 2009, Paper We7.5.6.

[7] M. J. O'Mahony, "Semiconductor laser optical amplifiers for use in future fiber systems," J. Lightw. Technol., vol. 6, no. 4, pp. 531-544, Apr. 1988.

[8] A. Garreau et al., "10 Gbit/s drop and continue colorless operation of a $1.5 \mu \mathrm{m}$ AlGaInAs reflective amplified electroabsorption modulator," in Proc. ECOC'06, Cannes, France, Sep. 2006, Paper We.1.6.5. 